s'imposer. Parti du Togo, où il avait analysé la parenté des peuples méridionaux avec les Yorouba de Nigéria, il a poursuivi par l'étude des Fang et des Foulbé au Cameroun et se dispose à étudier les migrations arabes au point où elles sont parvenues le plus au sud en Oubangui. Parvenu à ce terme de son voyage, en des lieux proches de la frontière du Soudan Anglo-Egyptien, M. de Pedrals se trouverait en état d'aborder la Nubie, un terrain d'extrême importance pour une compréhension de l'Afrique Noire et de son histoire. M. de Pedrals souhaiterait pouvoir se livrer à des recherches sur les traces possibles de la Napta légendaire qui aurait été le siège d'un Empire contemporain du premier royaume d'Égypte.

\title{
Exposition de bronzes et de terres cuites du Tchad (Cameroun et Afrique Equatoriale Francaise)
}

Sous le patronage du Ministre de la France d'Outre-mer et du Haut Commissaire de la République en Afrique Equatoriale Française, Annie Masson Detourbet et Jean-Paul Lebeuf ont exposé dernièrement à Paris les cent plus belles pièces qu'ils ont récoltées entre 1947 et r950 au cours de leurs deux dernières missions archéologiques dans la région du Tchad.

Ces témoins de la civilisation du $\operatorname{Tchad}\left(\mathrm{X}^{\mathrm{e}}\right.$ au XVI $\mathrm{X}^{\mathrm{e}}$ siècle) proviennent principalement du sanctuaire de 'Tago et des nécropoles de Midigué, anciens établissements sao. Les figurines de terre cuite se composent de représentations d'ancêtres 'divinisés' qui sont d'une facture particulièrement délicate, de figurations de danseurs masqués qui confirment l'existence, révélée par la tradition recueillie sur place, d'un corps de ballet rassemblé lors des fêtes religieuses, et de masques, simulacres d'ancêtres et d'enfants nouveau-nés, sortes d'ex-voto qui s'apparentent aux pierres représentatives d'ancêtres qui jouent un rôle important dans de nombreuses populations fétichistes d'Afrique noire. Le métal était représenté à cette exposition par des pièces de bronze plein coulé: colliers, anneaux de bras et de cheville, pectoral à trois branches, coupe à libations, gros anneaux qui eurent vraisemblablement un usage rituel, pendentifs zoomorphes.

\section{Mission d'étude des centres urbains des territoires du Tchad et de l'Oubangui-Chari}

Jean-Paul Lebeuf, Chargé de Recherches au Centre National de la Recherche Scientifique, et sa femme, Annie Masson Detourbet, chargés de mission par le Gouvernement Général de l'Afrique Equatoriale Française, ont accompli en I950 une étude préliminaire des Centres urbains de la Fédération pour le compte du Haut Commissaire, M. Cornut-Gentille.

Conformément à un plan d'ensemble dont les travaux de cette mission constituent la première partie, ils ont poursuivi à Fortlamy ('Territoire du Tchad) et à Bangui (Territoire de l'Oubangui-Chari) une enquête avec la collaboration des différents services administratifs de ces régions. Des rapports ont été remis au Haut Commissariat; ils feront l'objet de publications prochaines qui serviront de base à des études ultérieures.

Les principaux problèmes qui ont été abordés sont les suivants : création des villes et leur accroissement; influence des événements extérieurs (guerres mondiales, mandats sur le Cameroun, développement économique de la Fédération); aspect général des villes, types d'habitation, division en quartiers, organisation administrative; raisons de l'abandon du village et de la venue en ville; répartition des différents groupes ethniques, rassemblement et dispersion, évolution et accroissement du peuplement, chiffre de la population, groupes principaux, minorités autochtones et étrangères, inter-influence des groupes, mouvements de population; relations avec le milieu originel et persistance (ou abandon) des usages traditionnels; mariage, régime dotal et leur évolution au contact d'étrangers; associations d'Africains; les métiers et leur répartition suivant les populations; persistance, renouveau ou abandon des techniques anciennes; les métiers féminins; les langues véhiculaires et le problème de l'enseignement; la notion de nationalité; le problème religieux et les incidences 